\title{
Higgs decay into four leptons in the presence of dimension-six operators
}

\author{
Stefano Boselli*t \\ INFN, Sezione di Pavia, \\ Cavendish Laboratory, University of Cambridge \\ E-mail: stefano.boselliepv.infn.it
}

\begin{abstract}
A new study on the indirect effects of new physics in the Higgs decay into four charged leptons channel is reported. We study the impact of the deviations induced by the effective field theory dimension-six operators on few relevant angular observables, and we compare them with the contribution of the full electroweak corrections. The calculation is implemented in a new version of the event generator Hto 41 , which can provide predictions in terms of different EFT-bases and is available for data analyses at the LHC.
\end{abstract}

The European Physical Society Conference on High Energy Physics

5-12 July, 2017

Venice

\footnotetext{
* Speaker.

${ }^{\dagger}$ In collaboration with C. M. Carloni Calame, G. Montagna, O. Nicrosini, F. Piccinini and A. Shivaji [1]
} 


\section{Introduction}

The lack of direct evidence of new particles at the LHC seems to indicate that the scale of New Physics (NP) $\Lambda$ could be well separated from the energy range of the Standard Model (SM) spectrum. For this reason, the Standard Model Effective Field Theory (SMEFT) has become a phenomenological standard for the indirect searches of NP at the LHC, providing a model independent parametrization of NP effects at the electroweak (EW) scale. Once the heavy degrees of freedom have been integrated out, the effective Lagrangian takes the form

$$
\mathscr{L}_{\mathrm{EFT}}=\mathscr{L}_{\mathrm{SM}}+\frac{1}{\Lambda} \sum_{i} c_{i}^{(5)} \mathscr{O}_{i}^{(5)}+\frac{1}{\Lambda^{2}} \sum_{i} c_{i}^{(6)} \mathscr{O}_{i}^{(6)}+\cdots
$$

where $\mathscr{L}_{\mathrm{SM}}$ in Eq. (1.1) is the SM Lagrangian and it represents the lowest-order term of a series in the canonical dimension $D$. Each consecutive term is suppressed by larger powers of the NP scale $\Lambda$. Neglecting the $D=5$ lepton flavor violating operator [2], the leading Beyond-Standard-Model (BSM) effects are expected to be captured by $D=6$ operators.

In the Higgs sector the majority of SMEFT studies have interpreted the LHC data on Higgs production and decay modes to derive constraints on the $\mathrm{D}=6$ parameters.

The global analysis of signal strengths carried out in Ref. [3] with Run-I data leaves "blind directions" in the $D=6$ parameter space. In order to lift this degeneracy more data is needed and the study of differential distributions will provide a complementary piece of information. This motivates the efforts for the release of a new version of the Hto 4 l event generator [4], where new matrix elements for the Higgs decay into four leptons in the presence of $D=6$ operators have been implemented. The code is available for public use.

In my talk I describe some features of the updated version of the code and I present some numerical results for few observables that turned out to be particularly sensitive to the presence of anomalous couplings ${ }^{1}$.

\section{Computational details}

The computation of the matrix elements in presence of $D=6$ operators has been carried out in the so-called Higgs basis [13], which is constructed in terms of mass eigenstates and whose coefficients are connected to the Wilson coefficients of any other basis through linear transformations. There are four classes of $D=6$ operators giving rise to anomalous interactions in the $H \rightarrow 4 \ell$ decay channel. The first class includes five $\mathrm{CP}$-even and three $\mathrm{CP}$-odd operators giving rise to anomalous $H V V(V=\gamma, Z)$ couplings

\footnotetext{
${ }^{1}$ Other model independent NP studies in the $H \rightarrow 4 \ell$ channel have been carried out in Refs. [5, 6, 7, 8, 9, 10, 11, 12].
} 


$$
\begin{aligned}
& \mathscr{L}_{D=6}^{H V V}=\frac{H}{v}\left[\left(1+\delta c_{Z}\right) \frac{1}{4}\left(g_{1}^{2}+g_{2}^{2}\right) v^{2} Z_{\mu} Z^{\mu}+\right. \\
& +c_{\gamma \gamma} \frac{e^{2}}{4} A_{\mu v} A^{\mu v}+c_{Z \gamma} \frac{e \sqrt{g_{1}^{2}+g_{2}^{2}}}{2} Z_{\mu v} A^{\mu v}+c_{Z Z} \frac{g_{1}^{2}+g_{2}^{2}}{4} Z_{\mu v} Z^{\mu v}+ \\
& +c_{Z \square} g_{2}^{2} Z_{\mu} \partial_{v} Z^{\mu v}+c_{\gamma \square} g_{1} g_{2} Z_{\mu} \partial_{v} A^{\mu v}+ \\
& \left.+\tilde{c}_{\gamma \gamma} \frac{e^{2}}{4} A_{\mu v} \tilde{A}^{\mu v}+\tilde{c}_{Z \gamma} \frac{e \sqrt{g_{1}^{2}+g_{2}^{2}}}{2} Z_{\mu v} \tilde{A}^{\mu v}+\tilde{c}_{Z Z} \frac{g_{1}^{2}+g_{2}^{2}}{4} Z_{\mu v} \tilde{Z}^{\mu v}\right],
\end{aligned}
$$

Of the six CP-even couplings in Eq. (2.1) only five are independent. We choose $c_{\gamma \square}$ as dependent coupling, which is then expressed as the following linear combination:

$$
c_{\gamma \square}=\frac{1}{g_{2}^{2}-g_{1}^{2}}\left[2 g_{2}^{2} c_{Z \square}+\left(g_{2}^{2}+g_{1}^{2}\right) c_{Z Z}-e^{2} c_{\gamma \gamma}-\left(g_{2}^{2}-g_{1}^{2}\right) c_{Z \gamma}\right] .
$$

The second class of operators is given by the anomalous contributions to $Z \ell \ell$ vertex

$$
\begin{array}{r}
\mathscr{L}_{D=6}^{Z \ell \ell}=\sqrt{g_{1}^{2}+g_{2}^{2}} \sum_{\ell=e, \mu} Z_{\mu}\left[\bar{\ell}_{L} \gamma^{\mu}\left(I_{W, \ell}^{3}-s_{W}^{2} Q_{\ell}+\delta g_{L}^{Z \ell \ell}\right) \ell_{L}+\right. \\
\left.+\bar{\ell}_{R} \gamma^{\mu}\left(-s_{W}^{2} Q_{\ell}+\delta g_{R}^{Z \ell \ell}\right) \ell_{R}\right]
\end{array}
$$

while the third class gives rise to $H V \ell \ell$ contact interactions

$$
\mathscr{L}_{D=6}^{H Z \ell \ell}=2 \frac{\sqrt{g_{1}^{2}+g_{2}^{2}}}{v} \sum_{\ell=e, \mu}\left[\delta g_{L}^{H Z \ell \ell} H Z_{\mu} \bar{\ell}_{L} \gamma^{\mu} \ell_{L}+\delta g_{R}^{H Z \ell \ell} H Z_{\mu} \bar{\ell}_{R} \gamma^{\mu} \ell_{R}\right]
$$

In the Higgs basis, the $H V \ell \ell$ couplings are not independent:

$$
\delta g_{L}^{H Z \ell \ell}=\delta g_{L}^{Z \ell \ell} \quad \delta g_{R}^{H Z \ell \ell}=\delta g_{R}^{Z \ell \ell}
$$

The last two contributions involve dipole interactions between $Z$ bosons and leptons and the dipole contact interactions of the Higgs boson. These terms are proportional to lepton masses and in the $m_{\ell} \rightarrow 0$ limit can be safely neglected.

The new matrix elements for $H \rightarrow 2 e 2 \mu$ and $H \rightarrow 4 e / 4 \mu$ included in the new version of the HtO 4 I code have been carried out with FORM [14], and have been compared with the ones generated by MadGraph5@MC_NLO [15] for several phase-space points, finding excellent agreement. We have included the quadratic contributions coming from squaring the $D=6$ matrix elements, with the possibility to turn them off in the code. The code is provided with a dictionary allowing to switch from the Higgs basis to the commonly used Warsaw [16] and SILH [17, 18] basis. The two mappings are totally general and are not affected by the specific assumption of the Higgs basis ${ }^{2}$. 

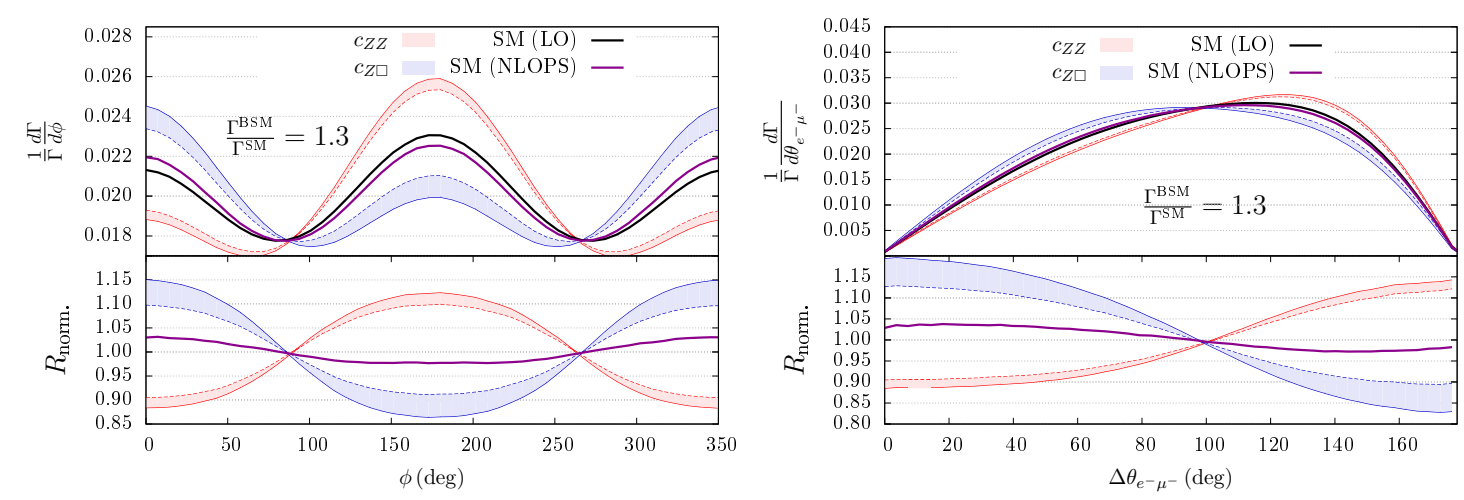

Figure 1: Upper Panels: normalized distributions for $\phi$ angle (left) and $\Delta \theta_{e_{-}} \mu_{-}$(right). Lower panels: Ratio of normalized distributions

\section{Numerical Results}

In this simple analysis we use the new version of Hto4l to simulate the decay of the Higgs boson at the differential level in presence of anomalous couplings. For the sake of simplicity we consider one parameter at a time while the remaining ones are artificially set to zero. We focus our attention on $c_{Z Z}$ and $c_{Z \square}$ which are poorly constrained by current data. To compare the characteristic effects of the coefficients we choose benchmark values giving rise to a $30 \%$ of excess in the $\Gamma(H \rightarrow 2 e 2 \mu)$ partial width. Of the observables taken into account, the two most sensitive ones are the angle $\phi$ between the decay planes of the two intermediate gauge-bosons the angle $\Delta \theta_{e_{-}} \mu_{-}$between the electron and the muon. Both angles are defined in the Higgs rest frame. In Fig. 1 we compare the BSM predictions for the normalized distributions of these observables with the SM ones at Leading Order (LO) and at Next-to-Leading Order EW accuracy matched to a QED Parton Shower (NLOPS in the following). Continuous lines in the plots refer to distributions obtained by considering only the effects of interference, while for the dashed ones quadratics effects have been also taken into account. We find that the effect of $c_{Z Z}$ and $c_{Z \square}$ is opposite in nature. The effects of quadratic terms depends on the considered observable and in general are not negligible.

\section{Conclusion}

The analysis presented in this talk seems to indicate that the negative correlation between $c_{Z Z}$ and $c_{Z \square}$ arising from the global analysis of the Run-I data [3] would not be completely removed by taking the angular observables defined above into account. Nevertheless, we are performing a phenomenological analysis by considering the statistical level reached with the HL-LHC in order to assess the improvements of $D=6$ operator constraints if differential information are taken into account ${ }^{3}$. We expect to present new results in the near future.

\footnotetext{
${ }^{2}$ For more details the reader should refer to Appendix B of Ref. [1] and to Ref. [19].

${ }^{3} \mathrm{~A}$ recent analysis in which angular asymmetries have been considered in the context of future leptonic colliders can be found in Ref. [20].
} 


\section{Acknowledgments}

SB would like to thank the organizer of the European Physical Society Conference on High energy physics and the conveners of the Higgs and New Physics session.

\section{References}

[1] S. Boselli, C. M. Carloni Calame, G. Montagna, O. Nicrosini, F. Piccinini, and A. Shivaji, Higgs decay into four charged leptons in the presence of dimension-six operators, arXiv:1703.06667.

[2] S. Weinberg, Baryon- and lepton-nonconserving processes, Phys. Rev. Lett. 43 (Nov, 1979) $1566-1570$.

[3] A. Falkowski, Effective field theory approach to LHC Higgs data, Pramana 87 (2016), no. 3 39, [arXiv:1505.00046].

[4] S. Boselli, C. M. Carloni Calame, G. Montagna, O. Nicrosini, and F. Piccinini, Higgs boson decay into four leptons at NLOPS electroweak accuracy, JHEP 06 (2015) 023, [arXiv: 1503.07394 ].

[5] D. Stolarski and R. Vega-Morales, Directly Measuring the Tensor Structure of the Scalar Coupling to Gauge Bosons, Phys. Rev. D86 (2012) 117504, [arXiv: 1208 . 4840].

[6] Y. Chen, N. Tran, and R. Vega-Morales, Scrutinizing the Higgs Signal and Background in the $2 e 2 \mu$ Golden Channel, JHEP 01 (2013) 182, [arXiv: 1211.1959 ].

[7] Y. Chen and R. Vega-Morales, Extracting Effective Higgs Couplings in the Golden Channel, JHEP 04 (2014) 057, [arXiv:1310.2893].

[8] M. Beneke, D. Boito, and Y.-M. Wang, Anomalous Higgs couplings in angular asymmetries of $H \rightarrow Z \ell^{+} \ell^{-}$and $e^{+} e^{-} \rightarrow H Z$, JHEP 11 (2014) 028, [arXiv:1406.1361].

[9] M. Gonzalez-Alonso, A. Greljo, G. Isidori, and D. Marzocca, Pseudo-observables in Higgs decays, Eur. Phys. J. C75 (2015) 128, [arXiv: 1412.6038$]$.

[10] Y. Chen, E. Di Marco, J. Lykken, M. Spiropulu, R. Vega-Morales, and S. Xie, 8D likelihood effective Higgs couplings extraction framework in $h \rightarrow 4 \ell$, JHEP 01 (2015) 125, [arXiv: 1401.2077 ].

[11] M. Gonzalez-Alonso, A. Greljo, G. Isidori, and D. Marzocca, Electroweak bounds on Higgs pseudo-observables and $h \rightarrow 4 \ell$ decays, Eur. Phys. J. C75 (2015) 341, [arXiv: 1504.04018 ].

[12] M. Bordone, A. Greljo, G. Isidori, D. Marzocca, and A. Pattori, Higgs Pseudo Observables and Radiative Corrections, Eur. Phys. J. C75 (2015), no. 8 385, [arXiv: 1507.02555$].$

[13] LHC Higgs Cross Section Working Group Collaboration, D. de Florian et al., Handbook of LHC Higgs Cross Sections: 4. Deciphering the Nature of the Higgs Sector, arXiv:1610.07922.

[14] J. Vermaseren, New features of FORM, math-ph/0010025.

[15] J. Alwall, R. Frederix, S. Frixione, V. Hirschi, F. Maltoni, O. Mattelaer, H. S. Shao, T. Stelzer, P. Torrielli, and M. Zaro, The automated computation of tree-level and next-to-leading order differential cross sections, and their matching to parton shower simulations, JHEP 07 (2014) 079, [arXiv:1405.0301].

[16] B. Grzadkowski, M. Iskrzynski, M. Misiak, and J. Rosiek, Dimension-Six Terms in the Standard Model Lagrangian, JHEP 10 (2010) 085, [arXiv: 1008 .4884].

[17] G. F. Giudice, C. Grojean, A. Pomarol, and R. Rattazzi, The Strongly-Interacting Light Higgs, JHEP 06 (2007) 045, [hep-ph/ 0703164$].$ 
[18] R. Contino, M. Ghezzi, C. Grojean, M. Muhlleitner, and M. Spira, Effective Lagrangian for a light Higgs-like scalar, JHEP 07 (2013) 035, [arXiv: 1303 . 3876].

[19] I. Brivio and M. Trott, Scheming in the SMEFT... and a reparameterization invariance!, JHEP 07 (2017) 148, [arXiv:1701.06424].

[20] G. Durieux, C. Grojean, J. Gu, and K. Wang, The leptonic future of the Higgs, JHEP 09 (2017) 014, [arXiv:1704.02333]. 